\title{
Observation on the Effect of Comfortable Nursing in ICU Patients with Severe Infection
}

Yexin $\mathbf{L i}^{*}$

Yemeng Tianshui Medical Co., Ltd. E-mail: yx@163.con

\begin{abstract}
Objective: this article aims to explore the effect of comfortable nursing in ICU patients with severe infection. Methods: from January 2018 to January 2019, 44 patients with severe infection were selected as research objects. They were randomly divided into observation group $(n=22)$ and control group $(n=22)$. The control group was given routine nursing, while the observation group was given comfortable nursing on the basis of the control group. Pittsburgh Sleep Quality Index (PSQ1), Self-rating Anxiety Scale (SAS) and the incidence of adverse reactions were observed and compared between the two groups before and after intervention. Results: the PSQI score and SAS score of the patients in the observation group were lower than those in the control group. The incidence of adverse reactions in the observation group was $4.59 \%$, which was significantly lower than that in the control group (27.27\%), and the difference between the two groups was statistically significant $(\mathrm{P}<0.05)$. Conclusion: comfortable nursing intervention for ICU patients with severe infection can obviously improve their sleep quality and negative emotions, and reduce the incidence of adverse reactions during hospitalization, which has clinical application value.
\end{abstract}

Keywords: Comfortable Nursing; ICU; Severe Infection

\section{Introduction}

Severe infection is a common clinical disease. Patients are often treated in intensive care unit (ICU) because they are seriously ill. ICU patients mainly come from emergency department, operating room and other departments of hospitals. Their ability to deal with the stimulus from the hospital stress environment is poor. Besides, ICU patients will have various physical and psychological discomfort due to the lack of family members' companionship. Therefore, how to control patients' infection symptoms and improve their treatment effect has always been the focus of intensive care medicine. In this study, 44 patients with severe infection treated from January 2018 to January 2019 were selected as the research objects, and the application effect of comfortable nursing in ICU patients with severe infection was discussed to achieve the purpose of promoting early rehabilitation of patients.

\section{Materials and methods}

From January 2018 to January 2019, 44 patients with severe infection admitted to the Department of Critical Care Medicine of Ninghe District Hospital, located in Tianjin, were taken as research objects, and were divided into observation group $(n=22)$ and control group ( $n=22)$ according to random number table method. In the control group, there were 12 males and 10 females with an average age of (40.5 6.4) years, ranging from 25 to 55 years old. There were 11 males and 11 females in

\footnotetext{
Copyright (C) 2020 Yexin Li

doi: $10.18686 /$ jn.v9i2.171

This is an open-access article distributed under the terms of the Creative Commons Attribution Non-Commercial License

(http://creativecommons.org/licenses/by-nc/4.0/), which permits unrestricted non-commercial use, distribution, and reproduction in any medium, provided the original work is properly cited.
} 
the observation group, with an average age of (39.8 6.2) years, ranging from 28 to 54 years old. There was no significant difference in gender and age between the two groups $(\mathrm{P}>0.05)$. This study was approved by the Medical Ethics Committee of the hospital, and the patients and their families signed the informed consent form. Inclusion criteria: those who need intensive care according to the diagnostic criteria of severe infection in Diagnosis and Treatment of Severe Infection; Those who are conscious and have normal intelligence. Patients are over 20 years old or; Those who sleep normally before admission. Exclusion criteria: those with severe mental organic diseases; Patients with tumor diseases are unwilling to participate in the investigation. 1.3 Nursing methods The patients in the control group were given routine nursing intervention, including routine vital signs monitoring, medication nursing, infection prevention, psychological nursing and prevention of related complications. Patients in the observation group were given comfortable nursing intervention, the main contents are as follows. Environmental nursing: keep the indoor temperature at $22{ }^{\circ} \mathrm{C} \sim 24{ }^{\circ} \mathrm{C}$ and humidity at $50 \% \sim 60 \%$, disinfect the indoor air of ICU regularly every day, and enhance the living atmosphere in ICU, so that the indoor layout tends to be life-oriented. Because the light in ICU is bright, attention should be paid to taking morning and evening light care for patients, and keeping reasonable light at night to avoid stimulation Studies have shown that the color of the ward will properly affect the psychological and physiological health of patients. Therefore, mild colors such as light green or blue should be used in the ICU room, which can obviously alleviate the negative effects such as headache, insomnia, fear and anxiety of patients. When there are other patients in the rescue state, pull up the curtain in time to reduce unnecessary psychological stimulation of patients. When conditions permit, you can listen to some music that is conducive to psychological relief and relieve the mental pressure of patients

Nursing staff should take care of patients in a full and positive mental state, and wash patients with warm water every morning and evening. When washing, they should move warmly, and at the same time, avoid the thinker from seeing the cold. Do a good job in oral care of patients, and use $2 \% \sim 3 \%$ NAHCO3 every morning and evening for oral care to prevent oral diseases. Do a good job in nursing nasal feeding, keep the food temperature at a proper temperature, feed it every $2 \mathrm{~h}$, wash the nasal feeding tube after feeding, and change the nasal feeding tube regularly. Do a good job in the whole body's pipeline nursing, and help patients to clamp the drainage tube regularly when turning over, so as to prevent the drainage tube from falling off due to turning over. According to the patient's condition, daily life operations such as manicuring nails, changing sheets, quilts, sick clothes, cleaning hair, etc. should be carried out in time to make the patient feel comfortable. When laying an air cushion bed for patients, be careful not to deflate too slowly and keep it at 34 . When the patient is awake, the nursing staff should take the initiative to explain the purpose and steps of the operation to the patient when doing every operation, and at the same time, the action should be gentle and agile to avoid adverse effects on the patient.

Good sleeping condition is an important factor to promote patients' early recovery to health. Patients with severe $1 \mathrm{CU}$ infection are in critical condition, so the room should be kept bright at any time, so as to better discover the changes of patients' condition. However, at the same time, the tightness and light condition in ICU will affect the rest of patients. Nursing staff should try their best to create a quieter and more comfortable sleeping environment for patients, such as reducing unnecessary light stimulation to patients, using bed curtains at rest, and doing good night care. If the condition allows, it can help patients soak their feet before going to bed, choose suitable pillows and bedding, assist patients to take appropriate sleeping position, and give appropriate sleeping and sedative drugs. At the same time, they should try their best to take centralized treatment during night treatment. Psychological comfort: the intensive care unit is a closed environment. The fear caused by the lack of family members' companionship and unfamiliar environment in the ICU, as well as the anxiety about their own illness, can easily cause patients to have negative emotions such as anxiety, depression, irritability and fear, which seriously affect the treatment effect. Nursing staff should try their best to meet the needs of patients according to their own psychological characteristics, provide patients 
with reading publications, display photos of relatives and familiar items, and allow family members to visit at fixed time every day. Popularize relevant CU knowledge to family members, assist family members to comfort patients, explain some successful cases of intensive care unit treatment for patients, build up the confidence of patients and their families to overcome diseases, encourage patients to actively treat and strive to overcome diseases as soon as possible.

Pittsburgh Sleep Quality Index (PSQI), Self-rating Anxiety Scale (SAS) and the incidence of adverse reactions were observed and compared between the two groups before and after intervention. PSQI Sleep Quality Score: It consists of 24 dimensions, with a score of $0 \sim 3$, with a total score of $0 \sim 2$. The higher the score, the worse the sleep quality of the patient. SASP score: it consists of 20 dimensions, and the total score is obtained by adding the scores of 20 dimensions and multiplying them by 1.25. The total score is 50 59 as mild anxiety, and 60 69 as moderate anxiety. More than 69 points are considered as severe anxiety. Observe whether the patients have adverse reactions such as respiratory failure, high fever, low blood pressure, worsening cold infection of limbs, failure of multiple organs of the whole body, and disturbance of consciousness. Incidence of adverse reactions $=$ number of cases with adverse reactions/total number of cases $\times 100 \%$. SPSS 22.0 was used for data analysis, PSQI score and SAS score were expressed by ( $\mathrm{x}$ s), and the comparison was conducted by test. The incidence of adverse reactions was expressed by (\%) and analyzed by $\mathrm{x} 2$ test, with $\mathrm{P}<0.05$ as the difference.

\section{Results}

The comparison of PSQI score and SAS score between the two groups before and after the intervention showed that the PSQI score and SAS score of the observation group were lower than those of the control group after the nursing intervention, and the difference between the two groups was statistically significant $(\mathrm{P} \leq 0.05)$. Comparing the incidence of adverse reactions between the two groups, 6 patients in the control group had adverse reactions during hospitalization in ICU, and the incidence of adverse reactions was $27.27 \%$; There was one case of adverse reaction in the observation group, and the incidence of adverse reaction was $4.55 \%$, with significant difference between the two groups ( $\mathrm{x} 2=4.247, \mathrm{P}=0.043$ ).

\section{Discussion}

ICU is a special ward in the hospital. Due to its closed treatment environment, more intensive care and invasive treatment, high indoor light intensity, and absence of family members and expensive treatment costs, the sleep quality of conscious patients with severe infection is affected. Patients of psychological barriers of different degrees occur, and thus the treatment effect is affected. However, some studies have shown that comfortable nursing intervention can significantly improve the negative emotions of patients with $\mathrm{CU}$, thus improving the therapeutic effect. Comfortable nursing mode is nursing intervention for patients from physiological, psychological and life aspects, and comfortable nursing can improve the sleep quality of patients by controlling indoor illumination; Through careful life care, patients feel at ease and comfortable; Through psychological comfort to the patients, they can relax their mood, increase their confidence in overcoming the disease, improve the treatment effect and promote their early recovery. The results of this study showed that the PSQI score and SAS score in the observation group were [(741 0.20), (4937 5.26)] lower than those in the control group [(10.67 1.53), (58.74 3.40) 1 , respectively. The incidence of adverse reactions in the observation group was $4.5 \%$, which was significantly lower than that in the control group (27.27\%), and there was significant difference between the two groups $(\mathrm{P}<0.05)$. The result shows that comfortable nursing intervention can't be ignored for patients with severe infection.

\section{Conclusion}

To sum up, the application of comfortable nursing intervention to ICU patients with severe infection can significantly improve their sleep quality and negative emotions. Incidence of adverse reactions is significantly reduced, which has important clinical significance. 


\section{References}

1. Li S. The effect of ICU quality nursing intervention in early postoperative ventilator treatment of children with congenital heart disease (in Chinese). Journal of Medical Theory and Practice 2019; 33(10): 1680-1681.

2. $\mathrm{Gu} \mathrm{C}$. The effect of bundle analgesia and sedation nursing on the incidence of delirium in patients with mechanical ventilation in intensive care unit (in Chinese). Medical theory and practice 2019; 33(10): 1686-1688.

3. Li W, Quan M, Wu H, et al. Nursing research progress of ICU patients leaving bed early (in Chinese). Journal of Nurse Training 2019; 35(10): 892-895.

4. $\mathrm{Hu} \mathrm{J}$. Effect of comfort nursing combined with health education on rehabilitation of patients with cor pulmonale (in Chinese). Forum of Primary Medical Sciences 2020; 24(18): 2642-2643.
5. Tao Q. Analysis of the intervention effect of comfort nursing on breast cancer patients undergoing chemotherapy (in Chinese). Medical Doctor 2020; (07): 128-129.

6. Zhang Q, Wu Z, Yu C. Influence of whole-course comfortable nursing on comfort and psychological state of patients undergoing nasobiliary drainage (in Chinese). Medical Theory and Practice 2020; 33(11): 1866-1867+1854.

7. Fang Y, Wang L. Discussion on the application value of comfortable nursing in elderly rehabilitation nursing (in Chinese). Electronic Journal of Practical Clinical Nursing 2020; 5(24): $1+28$.

8. Wang H. Analysis of the effect of comfortable nursing on patients with femoral neck fracture, reducing the incidence of complications and improving nursing satisfaction (in Chinese). Electronic Journal of Practical Clinical Nursing 2020; 5(24): 10 . 